\title{
Elevated circulating SST2 associated with subclinical atherosclerosis in newly diagnosed primary hypertension
}

\author{
Ihsan Ates ${ }^{1}$, Nihal Ozkayar ${ }^{2}$, Hale Ates ${ }^{3}$, Uğur Nadir Karakulak ${ }^{4}$, Oğuzhan Kursun ${ }^{5}$, Canan Topcuoglu ${ }^{6}$, \\ Bayram Inan ${ }^{1}$ and Nisbet Yilmaz ${ }^{1}$
}

The aims of this study were to measure the levels of interleukin-33 (IL-33) and soluble Suppression of Tumorigenicity 2 (sST2) in patients with newly diagnosed primary hypertension (HT) and to determine the relationship between carotid intima-media thickness (CIMT) and IL-33/sST2. Eighty-two patients with newly diagnosed primary HT and ninety healthy volunteers were included in the study. CIMT $\geqslant 0.9 \mathrm{~mm}$ was considered as significant for subclinical atherosclerosis. The SST2 levels of patients with primary HT were higher than those of the control group, whereas the IL-33 levels of these patients were much lower than those of the control group. The SST2 levels were higher in patients with subclinical atherosclerosis than in control subjects or patients with primary HT but not with subclinical atherosclerosis. In the primary HT group, SST2 had a positive correlation with CIMT, 24-h systolic-diastolic blood pressure, low-density lipoprotein and C-reactive protein, whereas SST2 had a negative correlation with the IL-33 level. A stepwise multivariable logistic regression analysis revealed that SST2 is an independent risk factor for subclinical atherosclerosis. Although the diagnostic predictive value of $\mathrm{HT}$ risk was determined as $>51.8 \mathrm{pg} \mathrm{I}^{-1}$ in the receiver operating characteristic curve analysis in respect of the SST2 level, the diagnostic predictive value for subclinical atherosclerosis risk was determined to be $>107.2 \mathrm{pg} \mathrm{I}^{-1}$. The SST2 level displays a positive correlation with atherosclerotic changes, and is an independent risk factor for subclinical atherosclerosis expressed as increased CIMT.

Hypertension Research (2016) 39, 513-518; doi:10.1038/hr.2016.16; published online 25 February 2016

Keywords: carotid intima-media thickness; chronic inflammation; essential hypertension; interleukin 1; T helper-1

\section{INTRODUCTION}

Hypertension (HT) is one of the most important known risk factors for atherosclerosis. HT can initiate the atherosclerotic process alone and can also accelerate the atherosclerotic process by contributing to other risk factors (hyperlipidemia, diabetes, smoking, etc.). Hypertrophy in vascular structures, which is caused by high blood pressure, and endothelial dysfunction, which appears in the advanced stage, appear to be the major risk factors involved in the pathogenesis of atherosclerosis. ${ }^{1}$ In other words, the hypertrophic response of the vascular smooth muscle to high blood pressure is one of the most important indicators of subclinical atherosclerosis. Therefore, intima-media thickness, which measures the increase in large vessels such as the carotid, is an important biomarker of subclinical atherosclerosis. $^{2}$

Atherosclerosis is a chronic disease in which all of the risk factors listed above, as well as the inflammatory process, have an active role from the beginning to the end. ${ }^{3}$ In cases of HT where endothelial dysfunction has not developed, inflammatory cells begin to be produced in the endothelium and smooth muscle owing to increased levels of angiotensin $\mathrm{II}^{4-7}$ after endothelial dysfunction develops, inflammatory cells have an active role in the pathogenesis of atheromatous plaques. ${ }^{8}$ The fact that inflammatory cells have a critical role in the early stages of atherosclerosis and in the process of atheromatous plaque development suggests that some inflammatory markers could be predictive biomarkers of subclinical atherosclerosis. ${ }^{9,10}$ Recent studies have shown that inflammatory markers such as the highly sensitive C-reactive protein (CRP), ${ }^{9}$ interleukin $6^{11}$ and tumor necrosis factor $\alpha^{12,13}$ can predict atherosclerosis.

Another inflammatory marker that has been shown to be associated with cardiovascular disease is Suppression of Tumorigenicity 2 (ST2). ST2 is a receptor of interleukin-33 (IL-33) that is associated with Th-2 and is a member of the IL-1 receptor family. ${ }^{14}$ ST2 has two forms: soluble ST2 (sST2) and transmembrane ST2. ${ }^{15}$ The form that has been

\footnotetext{
${ }^{1}$ Department of Internal Medicine, Ankara Numune Training and Research Hospital, Ankara, Turkey; ${ }^{2}$ Department of Nephrology, Ankara Numune Training and Research Hospital, Ankara, Turkey; ${ }^{3}$ Department of Immunology and Allergy, Atatürk Chest Diseases and Thoracic Surgery Training and Research Hospital, Ankara, Turkey; ${ }^{4}$ Department of Cardiology, Ankara Occupational Diseases Hospital, Ankara, Turkey; ${ }^{5}$ Department of Neurology, Ankara Numune Training and Research Hospital, Ankara, Turkey and ${ }^{6}$ Department of Biochemistry, Ankara Numune Training and Research Hospital, Ankara, Turkey

Correspondence: Dr I Ates, Department of Internal Medicine, Ankara Numune Training and Research Hospital, 06100, Sihhiye, Ankara, Turkey.

E-mail: dr.ihsanates@hotmail.com

Received 18 November 2015; revised 22 December 2015; accepted 28 December 2015; published online 25 February 2016
} 
most frequently investigated in cardiovascular diseases is sST2. ${ }^{16}$ By binding to IL-33, sST2 causes a decrease in levels of IL-33, which has cardioprotective effects. ${ }^{17}$ Therefore, in previous studies, sST2 has been shown to be associated with coronary artery disease, ${ }^{15}$ myocardial infarction ${ }^{18}$ and ischemic heart disease. ${ }^{19}$

IL-33 is a member of the IL-1 family. IL-33 is known to be a cardioprotective marker, because it inhibits cardiomyocyte apoptosis and reduces myocardial fibrosis and myocyte hypertrophy. ${ }^{17,20,21}$ In addition, in animal models, atherosclerotic lesions shrink in the aortic sinus after exogenous IL-33 administration, suggesting that IL-33 is also an anti-atherosclerotic agent. ${ }^{20}$ Although the association of IL-33/ST2 with cardiovascular diseases and atherosclerosis is often discussed in the literature, no studies have investigated the association between IL-33/ST2 and asymptomatic organ damage (known as subclinical atherosclerotic changes) caused by HT.

On the basis of the above information, the aims of this study were to measure the IL-33 and sST2 levels in patients with newly diagnosed primary HT and to investigate the relationship between IL-33/sST2 and carotid intima-media thickness (CIMT), which is an important indicator of subclinical atherosclerosis.

\section{METHODS}

\section{Study population, design and setting}

This study used a cross-sectional design and was conducted in Ankara Numune Training and Research Hospital, Clinic of Internal Medicine between January and June 2015. Eighty-two patients ( 25 males and 57 females) over the age of 18 with newly diagnosed primary HT and who had not yet started treatment and 90 healthy volunteers (39 males and 51 females) without any known disease were included in the study. Primary HT patients were patients who attended the polyclinic with hypertensive symptoms, whose blood pressure measurements were determined to be high, and who had no secondary HT based on physical examination and laboratory findings. The control group consisted of 90 healthy volunteers with a normal mean 24-h blood pressure; the volunteers had attended the polyclinic for a check-up without having any known chronic disease or drug use. The demographic and characteristic features of the patients in the control group were obtained from the patients' files and patients' anamneses. Their body mass index (BMI) values were calculated by dividing the body dry weight by the square of the body height in meters $\left(\mathrm{BMI}=\mathrm{kg} \mathrm{m}^{-2}\right)$.

Patients with known cardiovascular, cerebrovascular or peripheral artery disease, diabetes, acute-chronic kidney or liver disease, or infective or rheumatic inflammatory disease were excluded from the study, as were those who smoked or took alcohol or regular medication. This study was designed in accordance with the 2013 Brasil version of the Helsinki Declaration, and was approved by the local Research Ethics Committee. All the participants provided written informed consent before the study.

\section{Ambulatory blood pressure monitoring}

For 24-h ambulatory blood pressure monitoring (ABPM), all participants wore a WatchBP 03 ABPM device (Microlife WatchBP AG, Widnau, Switzerland). The ABPM device was set to take 30-min measurements. The patients were allowed to continue their daily activities. Information necessary for the ABPM device to take accurate measurements was conveyed to the participants. As a result of the ABPM measurements, 24-h systolic blood pressure (SBP) and 24-h diastolic blood pressure (DBP) measurements were also obtained. Patients with mean 24 -h blood pressure $\geqslant 130 / 80 \mathrm{~mm} \mathrm{Hg}$ were considered to have primary HT. However, patients with mean 24-h blood pressure $<130 / 80 \mathrm{~mm} \mathrm{Hg}$ were considered to have normal blood pressure. The method was considered reliable if $>70 \%$ of the measurements were valid.

\section{CIMT measurement}

Carotid Doppler ultrasonography was performed by an expert neurosonologist who was blinded to the clinical data of the patients. For carotid artery ultrasonography, patients were asked to lie down on the examination table in the supine position and rotate their neck slightly to the opposite side. Measurements were taken with a high-resolution B-mode ultrasound device (Xario, Toshiba, Japan) using a 7.5-MHz linear array transducer. Measurements were obtained from three different plaque-free areas for each individual carotid artery: the proximal carotid, distal carotid and proximal internal carotid artery. Longitudinal measurements were taken from the distances defined by the vessel lumen echogenicity and media-adventitia echogenicity at the posterior wall of each area. For each carotid (left and right), the mean of the measurements was taken. CIMT $\geqslant 0.9 \mathrm{~mm}$ was considered to represent subclinical atherosclerosis or asymptomatic organ damage. ${ }^{22}$

\section{Laboratory parameters}

Venous blood samples were drawn from all participants between 0800 and $1000 \mathrm{~h}$ after $8 \mathrm{~h}$ of fasting. The blood samples were then centrifuged for $10 \mathrm{~min}$ at 4000 r.p.m., and the serum and plasma samples were separated. The serum samples were stored at $-80^{\circ} \mathrm{C}$ before the sST2, IL-33, lipid parameters and CRP levels were measured.

CRP was measured using the immunoturbidimetric method, and the total cholesterol and triglyceride levels were measured using the enzymatic colorimetric method. High-density lipoprotein (HDL) cholesterol was measured using the homogeneous enzymatic colorimetric method in a Hitachi Modular P800 (Roche Diagnostics, Indianapolis, IN, USA) auto analyzer. Low-density lipoprotein (LDL) cholesterol was calculated using the Friedewald method. $^{23}$

The serum IL-33 level was measured using a commercial ELISA kit Bioscience made by the Affymetrix Company, Vienna, Austria (REF No: BMS2048, LOT No: 107276014). The sensitivity of the assay was $0.2 \mathrm{pg} \mathrm{l}^{-1}$. The intra-study coefficient of variation (CV\%) was $4.7 \%$, and the and inter-study CV\% was $6.9 \%$.

The serum sST2 level was measured using a human IL-1 R4/ST2 commercial ELISA kit (Raybio, Raybiotech, Norcross, GA, USA, Cat No: ELH-IL-1 R4, LOT No: 022015 0296). The upper limit that the Rybio human ELISA kit could detect is $1200 \mathrm{pg}^{-1}$, and the lower limit is $2 \mathrm{pg}^{1^{-1}}$. The intra-study CV\% was $<10 \%$, and the inter-study CV\% was $<12 \%$.

\section{Statistical analysis}

The Statistical Package for Social Sciences (SPSS) for Windows 20 (IBM SPSS, Chicago, IL, USA) program was used for the statistical assessments. The Kolmogorov-Smirnov test was used to determine the distribution of the data. Continuous variables with normal distribution were expressed as the mean \pm s.d., and continuous variables without normal distribution were expressed as the median (interquartile range). Categorical variables were presented as numbers and percentages. Continuous variables were compared with an independent sample $t$-test or Mann-Whitney $U$-test where appropriate, and the Bonferroni correction was applied to the two sub-group comparisons. The relationships between the numeric parameters were analyzed by Pearson and Spearman correlation analyses. A stepwise linear regression analysis was used to identify independent predictors of the CIMT and sST2 levels. Logarithmic transformation was applied to the sST2, CIMT, triglycerides and CRP levels before the regression analysis. A stepwise logistic regression analysis was used to identify independent predictors of subclinical atherosclerosis risk. A receiver operating characteristic curve analysis and the Youden index method were used to calculate the predictive values of the SST2 level for primary HT and subclinical atherosclerosis risk. The diagnostic predictive value with the highest Youden index level was considered to be the best diagnostic predictive value. $P<0.05$ was considered as significant for the statistical analyses.

\section{RESULTS}

Table 1 summarizes the demographic characteristics and laboratory findings of the study population. The age and sex ratios were similar in the primary HT and control groups $(P>0.05)$. The BMI was high in the primary HT group compared with the control group $\left(30 \pm 6 \mathrm{~kg} \mathrm{~m}^{-2}\right.$ and $26 \pm 4 \mathrm{~kg} \mathrm{~m}^{-2}$, respectively; $\left.P<0.001\right)$. The mean 24-h SBP and DBP levels $(148 \pm 18 \mathrm{~mm} \mathrm{Hg}$ and $113 \pm 8 \mathrm{~mm} \mathrm{Hg}$; $P<0.001 ; 89 \pm 9 \mathrm{~mm} \mathrm{Hg}$ and $74 \pm 8 \mathrm{~mm} \mathrm{Hg}$, respectively; $P<0.001)$ 
Table 1 Demographic characteristics and laboratory findings of study population

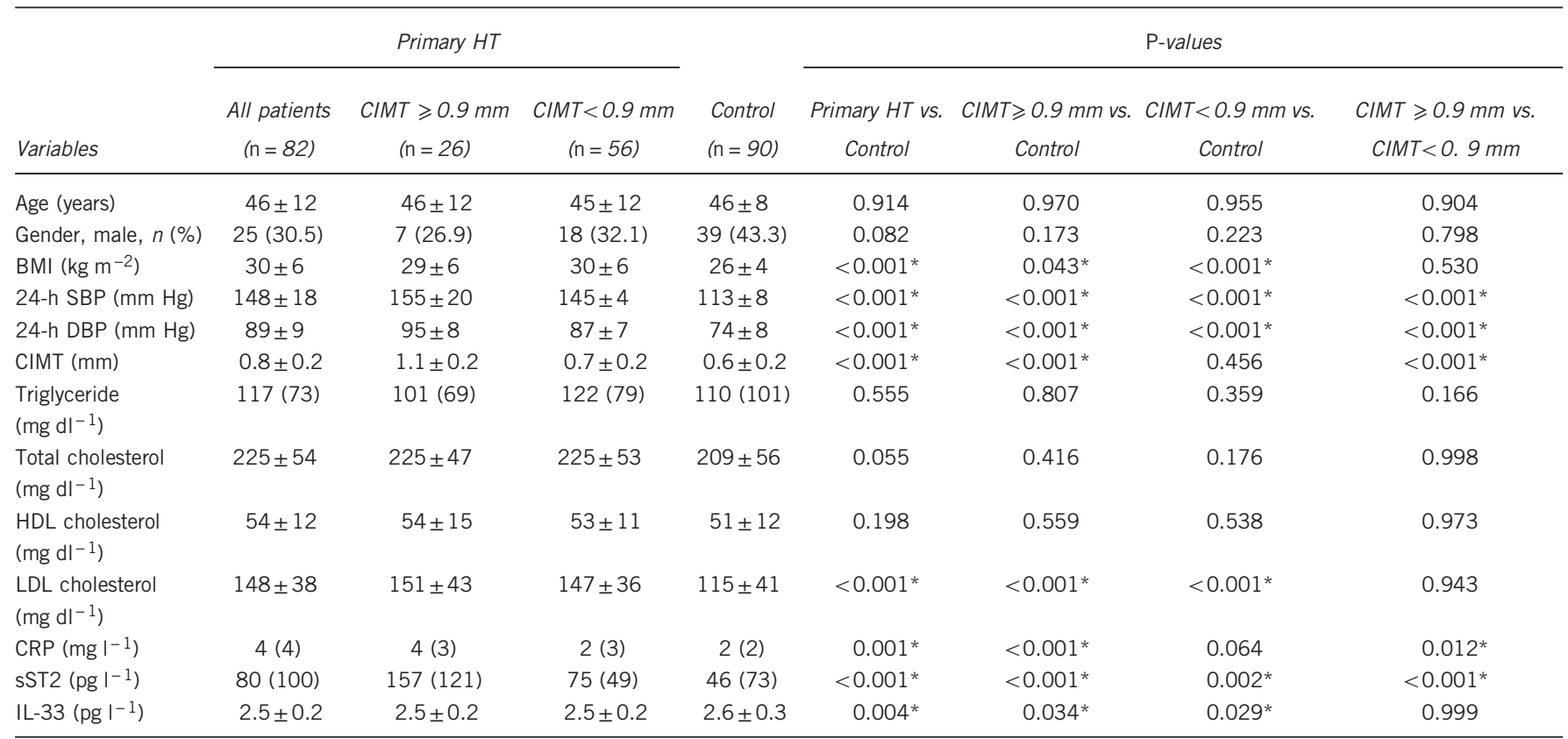

Abbreviations: BMI, body mass index; CIMT, carotid intima-media thickness; CRP, C-reactive protein; DBP, diastolic blood pressure; HDL, high-density lipoprotein; HT, hypertension;

IL-33, interleukin-33; LDL, low-density lipoprotein; SBP, systolic blood pressure; SST2, soluble suppression of tumorigenicity 2.

${ }^{*} P<0.05$ indicates statistical significance. In dual comparison of normally distributed numerical variables, Bonferroni-corrected $t$-test was applied, in dual comparison of numerical variables that do not display normal distribution, Bonferroni-corrected Mann-Whitney U-test was applied.

were high in the primary HT group compared with the control group. The mean total cholesterol was high in the primary HT group compared with the control group, and the statistical significance was borderline $\left(225 \pm 54 \mathrm{mg} \mathrm{dl}^{-1}\right.$ and $209 \pm 56 \mathrm{mg} \mathrm{dl}^{-1}$, respectively; $P=0.055)$. The mean LDL cholesterol level was also significantly higher in patients with primary HT $\left(148 \pm 38 \mathrm{mg} \mathrm{dl}^{-1}\right.$ and $115 \pm 41 \mathrm{mg} \mathrm{dl}^{-1}$, respectively; $P<0.001$ ).

The median primary sST2 level was high in HT patients than in control subjects ( 80 and $46 \mathrm{pgl}^{-1}$, respectively; $P<0.001$ ), and the mean IL-33 value was low compared with the control group $\left(2.5 \pm 0.2 \mathrm{pgl}^{-1}\right.$ and $2.6 \pm 0.3 \mathrm{pgl}^{-1}$, respectively; $\left.P=0.004\right)$. The mean CIMT level was also significantly higher in patients with primary HT than in control subjects $(0.8 \pm 0.2 \mathrm{~mm}$ and $0.6 \pm 0.2 \mathrm{~mm}$, respectively; $P<0.001$ ).

In patients with subclinical atherosclerosis, the BMI, 24-h SBP, 24-h DBP, LDL, CRP, CIMT and sST2 levels were higher than in the control group, whereas the IL-33 level was lower. Among patients with primary HT, the CRP and sST2 levels were significantly higher in patients who had subclinical atherosclerosis than in those who did not (Table 1).

The correlation analysis between CIMT, sST2, IL-33 and other demographic and laboratory findings in the primary HT patient group is shown in Table 2. The CIMT displayed a positive correlation with sST2 $(r=0.733, P<0.001)$ (Figure 1$), 24-\mathrm{h}$ SBP $(r=0.305, P<0.001)$, 24-h DBP $(r=0.300, P<0.001)$, triglyceride $(r=0.294, P=0.011)$, LDL cholesterol $(r=0.393, P<0.001)$ and CRP $(r=0.286, P<0.001)$, and a negative correlation with IL-33 $(r=0.367, P=0.003)$. The sST2 level displayed a positive correlation with 24-h SBP $(r=0.308$, $P=0.010), \quad 24-\mathrm{h}$ DBP $(r=0.370, \quad P<0.001), \quad$ LDL cholesterol $(r=0.347, P<0.001)$ and $\operatorname{CRP}(r=0.414, P<0.001)$, and a negative correlation with IL-33 $(r=0.354, P=0.013)$. The IL-33 level displayed a negative correlation with LDL cholesterol $(r=0.397, P=0.010)$ and 24-h DBP $(r=-0221, P=0.004)$.
Table 2 Demographic and clinical findings regarding CIMT, sST2 and IL-33 levels in the primary HT group

\begin{tabular}{|c|c|c|c|c|}
\hline \multirow[b]{2}{*}{ Variables } & \multicolumn{2}{|c|}{ sST2 } & \multicolumn{2}{|c|}{ IL-33 } \\
\hline & $r$ & $P$ & $r$ & $P$ \\
\hline CIMT & 0.733 & $<0.001^{*}$ & -0.367 & $0.003^{*}$ \\
\hline sST2 & - & - & -0.354 & $0.013^{*}$ \\
\hline IL-33 & -0.354 & $0.013^{*}$ & - & - \\
\hline Age & -0.011 & 0.882 & -0.045 & 0.556 \\
\hline BMI & 0.082 & 0.287 & -0.061 & 0.425 \\
\hline 24-h SBP & 0.308 & $0.010 *$ & -0.118 & 0.124 \\
\hline 24-h DBP & 0.370 & $<0.001^{*}$ & -0.221 & $0.004 *$ \\
\hline Triglyceride & -0.142 & 0.064 & -0.022 & 0.778 \\
\hline Total cholesterol & 0.032 & 0.676 & -0.088 & 0.257 \\
\hline HDL cholesterol & 0.131 & 0.090 & 0.019 & 0.801 \\
\hline LDL cholesterol & 0.347 & $<0.001 *$ & -0.397 & $0.010 *$ \\
\hline CRP & 0.414 & $<0.001 *$ & -0.033 & 0.671 \\
\hline
\end{tabular}

Abbreviations: BMI, body mass index; CIMT, carotid intima-media thickness; CRP, C-reactive protein; DBP, diastolic blood pressure; HDL, high-density lipoprotein; HT, hypertension; IL-33, interleukin-33; LDL, low-density lipoprotein; SBP, systolic blood pressure; sST2, soluble Suppression of Tumorigenicity 2.

${ }^{*} P<0.05$ indicates statistical significance.

The independent predictors of CIMT, sST2 and the risk of subclinical atherosclerosis are shown in detail in Table 3. The independent predictors that predicted the $\log (\mathrm{CIMT})$ level were $\log (\mathrm{sST} 2)(\beta \pm$ s.e. $=1.922 \pm 0.189 ; \quad P<0.001), \quad 24$-h $\quad$ SBP $\quad(\beta \pm$ s.e. $=1.595 \pm 0.527$; $P=0.003)$ and LDL cholesterol $(\beta \pm$ s.e. $=0.905 \pm 0.391 ; P=0.022)$, whereas the independent predictors of $\log (\mathrm{sST} 2)$ level were $\log ($ CIMT $) \quad(\beta \pm$ s.e. $=1.900 \pm 0.180 ; \quad P<0.001)$ and $\log (\mathrm{CRP}) \quad(\beta \pm$ s.e. $=0.129 \pm 0.027 ; P<0.001)$, and the independent risk factor of subclinical atherosclerosis was sST2 (odds ratio $=1.160 ; P=0.001$ ). 


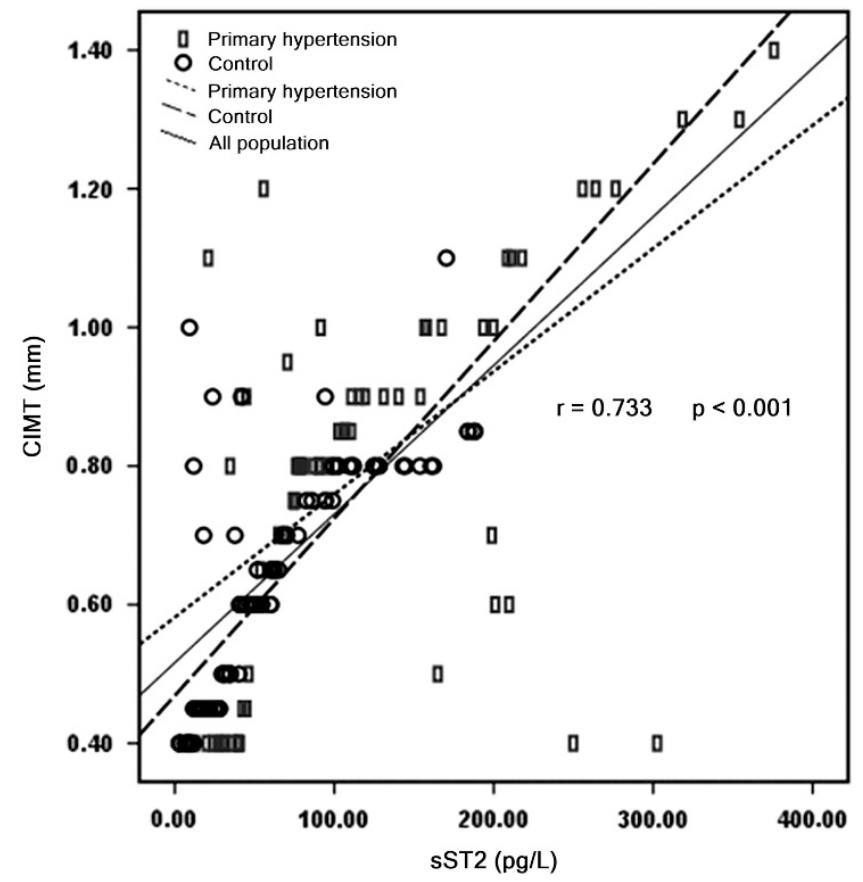

Figure 1 The correlation between CIMT and SST2 level shown on a scatter plot.

The diagnostic predictive value for primary HT risk was $>51.8 \mathrm{pg}^{-1}$ (area under curve \pm s.e. $=0.717 \pm 0.039 ; P<0.001$; sensitivity $=79.3 \%$, specificity $=58.9 \%$ ) on the receiver operating characteristic curve analysis of sST2 level, and the diagnostic predictive value for subclinical atherosclerosis risk the value was $>107.2 \mathrm{pg} \mathrm{l}^{-1}$ (area under curve \pm s.e. $=0.795 \pm 0.061 ; P<0.001$; sensitivity $=76.9 \%$, specificity $=87.5 \%)($ Figure 2$)$.

\section{DISCUSSION}

In this study, the median sST2 level in patients with newly diagnosed primary HT was higher than that in the control group, and the mean IL-33 level was lower. In addition, a positive correlation was discovered between sST2 levels and cardiovascular risk factors such as 24-h SBP and DBP, CRP, LDL cholesterol and CIMT. Moreover, in this patient group, sST2 was found to be an independent predictor of subclinical atherosclerosis.

In experimental studies, IL-33 has been shown to improve cardiac function and to contribute to cardiac cell survival, especially in post-MI patients, by inhibiting apoptosis in myocardial cells. ${ }^{24}$ sST2, when combined with IL-33, which is an important cardioprotective molecule in acute and chronic diseases, impedes the realization of the effects of IL-33. ${ }^{20}$ In previous studies, sST2 levels were found to be increased in coronary artery disease, acute myocardial infarction and coronary failure. ${ }^{15,18,25}$

Although the role of IL-33/sST2 in cardiovascular diseases is well known, its relationship with subclinical atherosclerosis is not clear. In our study, the IL-33 level was low and the sST2 level was high in patients with primary HT compared with the control group. In the correlation analysis, a positive correlation was found between sST2 and vascular and metabolic risk parameters such as 24-h SBP, 24-h DBP, LDL cholesterol, CIMT and CRP. In the regression analysis, CRP, which is an inflammatory marker, and CIMT, which is a vascular indicator, were shown to predict sST2. Therefore, sST2
Table 3 The determination of independent predictors for CIMT, sST2 and CIMT $\geqslant 9 \mathrm{~mm}$ with stepwise regression analysis

\begin{tabular}{lllll}
\hline & & \multicolumn{2}{c}{$95 \% \mathrm{Cl}$} & \\
\cline { 3 - 4 } & & & \\
Variables & $\beta \pm$ s.e. & Lower & Upper & $\mathrm{P}$ \\
\hline CIMT & & & & \\
$\quad$ SST2 & $1.922 \pm 0.189$ & 1.549 & 2.296 & $<0.001^{*}$ \\
24-h SBP & $1.595 \pm 0.527$ & 0.555 & 2.635 & $0.003^{*}$ \\
LDL cholesterol & $0.905 \pm 0.391$ & 0.132 & 1.678 & $0.022^{*}$ \\
$R^{2}=0.476 ; P<0.001^{*}$ & & & & \\
& & & & \\
sST2 & & & & \\
CIMT & $1.990 \pm 0.180$ & 1.634 & 2.345 & $<0.001^{*}$ \\
CRP & $0.129 \pm 0.027$ & 0.077 & 0.182 & $<0.001^{*}$
\end{tabular}

$R^{2}=0.553 ; P<0.001$ *

$\mathrm{OR}$

$C I M T \geqslant 9 \mathrm{~mm}^{\mathrm{c}}$

SST2 1.160

Nagelkerke $R^{2}=0.321 ; P<0.001^{*}$

$1.065 \quad 1.264$

$0.001^{*}$

Abbreviations: $\beta \pm$ s.e., non-standardized regression number \pm s.e.; $\mathrm{BMI}$, body mass index; $\mathrm{Cl}$, confidence intervals; CIMT, carotid intima-media thickness; CRP, C-reactive protein; DBP, diastolic blood pressure; IL-33, interleukin-33; LDL, low-density lipoprotein; OR, odds ratio; SBP, systolic blood pressure; SST2, soluble Suppression of Tumorigenicity 2.

${ }^{*} P<0.05$ indicates statistical significance. Before regression analysis, logarithmical transformation was performed on SST2, CIMT, triglyceride and CRP levels.

aStepwise multivariable linear regression analysis was used. Regression model age, gender, BMI, 24-h SBP, 24-h DBP, laboratory findings and log (sST2) and IL-33 were composed of risk factors.

bStepwise multivariable linear regression analysis was used. Regression model age, gender, BMI, 24-h SBP, 24-h DBP, laboratory findings and CIMT were composed of risk factors.

'Stepwise multivariable logistic regression analysis was used. Regression model age, gender, BMI, 24-h SBP, 24-h DBP, laboratory findings and sST2 and IL-33 were composed of risk factors.

is a versatile marker of vascular, atherogenic and metabolic characteristics.

Previous studies indicated a positive correlation between SBP and sST2. ${ }^{26,27}$ In these studies, it was discovered that stress caused by high blood pressure increased sST2 protein secretion from endothelial cells. Similarly, the study by Demyanets et al. ${ }^{28}$ showed that cardiac, aortic and coronary endothelial cells produced SST2. In a different study, it was shown that circulating IL-33 increases as a result of increasing myocardial pressure caused by HT. ${ }^{29}$ It is possible that as a response to the stress-induced increase in IL-33 levels, sST2 levels increase and reduce IL-33 levels by binding free IL-33 molecules. Increased production of reactive sST2 and IL-33 binding would change the balance of sST2/IL-33 in favor of sST2. In our study, the determination of a negative correlation between IL-33 and sST2 levels also supports this hypothesis.

Our study is the first to show that sST2 is an independent predictor of CIMT. The mechanistic explanation for the relationship between sST2 and CIMT could include several possibilities. The secretion of IL-33 into the circulation increases when endothelial cells are subjected to mechanical stress caused by an increase in systemic blood pressure. ${ }^{30,31}$ As a response to increasing IL-33 levels, the sST2 level would also begin to rise, and this increase would block the antihypertrophic and atheroprotective effects of IL-33. ${ }^{15}$ Moreover, it has been shown that sST2 can be secreted directly from the endothelial cells of vascular structures such as the aorta. ${ }^{28}$ In this case, increased CIMT due to high blood pressure becomes a significant source of sST2. sST2, which is a stimulant for atheroma plaques and has been associated with various atherosclerotic risk factors, is a risk factor for CIMT, and increased CIMT is a resource for SST2; this relationship explains the dual interaction and association between 

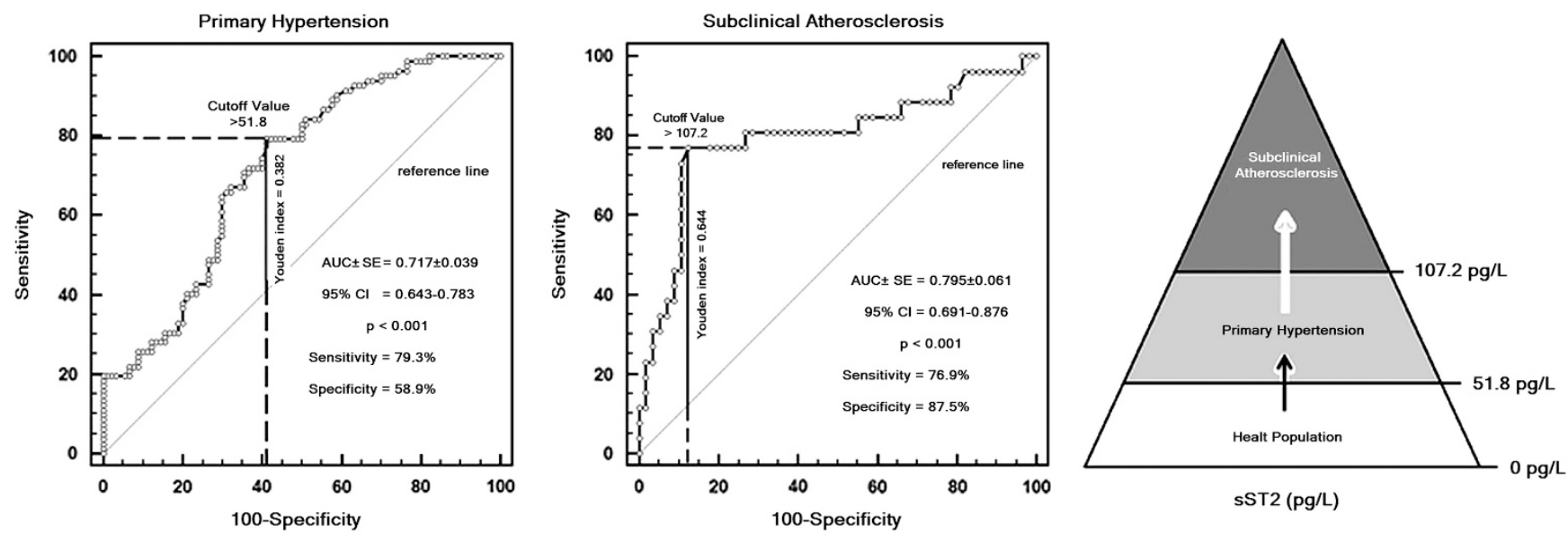

ROC analysis of sST2 (pg/L) for detection of cutoff value. Cutoff values were determined from the Youden index sST2: soluble suppression of tumorigenicity, AUC: area under the curve, Cl: confidence interval, SE:standart error

Figure 2 Determination of the diagnostic predictive value of SST2 for the risk of primary hypertension and subclinical atherosclerosis by receiver operating characteristic (ROC) curve analysis.

CIMT and sST2. This study and previous studies have shown that sST2 and CIMT are two separate atherogenic and vascular parameters that are effective in increasing the levels of each other. In addition to important clinical, metabolic and inflammatory markers including BMI, 24-h SBP, 24-h DBP, lipid profile and CRP, sST2 has been identified as an independent risk factor for subclinical atherosclerosis represented by increased CIMT, as shown by regression analysis.

The main limitations of our study are its cross-sectional design and the lack of follow-up in terms of the cardiovascular outcomes of patients. There is a need for a prospective study with a higher number of study subjects and a need for investigations of the association between sST2 and CIMT with prospective randomized controlled studies. Additionally, understanding how this association would respond to both HT and atherosclerosis would lead to a better understanding of the mechanism.

This study showed that in patients with primary HT, the IL-33 level was low and sST2 was high compared with control subjects, the sST2 level displayed a positive correlation with atherosclerotic changes, and sST2 is an independent risk factor for subclinical atherosclerosis represented by increased CIMT.

\section{CONFLICT OF INTEREST}

The authors declare no conflict of interest.

1 Alexander RW. Theodore Cooper Memorial Lecture. Hypertension and the pathogenesis of atherosclerosis. Oxidative stress and the mediation of arterial inflammatory response: a new perspective. Hypertension 1995; 25: 155-161.

2 Bauer M, Caviezel S, Teynor A, Erbel R, Mahabadi AA, Schmidt-Trucksass A. Carotid intima-media thickness as a biomarker of subclinical atherosclerosis. Swiss Med Weekly 2012; 142: w13705

3 Munro JM, Cotran RS. The pathogenesis of atherosclerosis: atherogenesis and inflammation. Lab Invest 1988; 58: 249-261.

4 Griendling KK, Ushio-Fukai M, Lassegue B, Alexander RW. Angiotensin II signaling in vascular smooth muscle. New concepts. Hypertension 1997; 29: 366-373.

5 Tummala PE, Chen XL, Sundell CL, Laursen JB, Hammes CP. Alexander RW, Harrison DG, Medford RM. Angiotensin II induces vascular cell adhesion molecule-1 expression in rat vasculature: a potential link between the renin-angiotensin system and atherosclerosis. Circulation 1999; 100: 1223-1229.

6 Heijnen BF, Van Essen H, Schalkwijk CG, Janssen BJ, Struijker-Boudier HA. Renal inflammatory markers during the onset of hypertension in spontaneously hypertensive rats. Hypertens Res 2014; 37: 100-109.
7 Kim HY, Cha HJ, Kim HS. CCL5 upregulates IL-10 expression and partially mediates the antihypertensive effects of $\mathrm{IL}-10$ in the vascular smooth muscle cells of spontaneously hypertensive rats. Hypertens Res 2015; 38: 666-674.

8 Libby P, Ridker PM, Maseri A. Inflammation and atherosclerosis. Circulation 2002; 105: 1135-1143.

9 Momiyama Y, Kawaguchi A, Kajiwara I, Ohmori R, Okada K, Saito I, Konishi M, Nakamura M, Sato S, Kokubo Y, Mannami T, Adachi H, Kario K, Iso H, Ohsuzu F, Tsushima M. Prognostic value of plasma high-sensitivity C-reactive protein levels in Japanese patients with stable coronary artery disease: the Japan NCVC-Collaborative Inflammation Cohort (JNIC) Study. Atherosclerosis 2009; 207: 272-276.

10 Kusunoki H, Taniyama Y, Otsu R, Rakugi H, Morishita R. Anti-inflammatory effects of hepatocyte growth factor on the vicious cycle of macrophages and adipocytes. Hypertens Res 2014; 37: 500-506.

11 Zhang B, Wang J, Xu Y, Zhou X, Liu J, Xu J, Li B, Zhi G. Correlative association of interleukin-6 with intima media thickness: a meta-analysis. Int J Clin Exp Med 2015; 8: 4731-4743

12 Edsfeldt A, Grufman H, Asciutto G, Nitulescu M, Persson A, Nilsson M, Nilsson J, Goncalves I. Circulating cytokines reflect the expression of pro-inflammatory cytokines in atherosclerotic plaques. Atherosclerosis 2015; 241: 443-449.

13 Bjorkbacka H, Fredrikson GN, Nilsson J. Emerging biomarkers and intervention targets for immune-modulation of atherosclerosis - a review of the experimental evidence. Atherosclerosis 2013; 227: 9-17.

14 Willems S, Quax PH, de Borst GJ, de Vries JP, Moll FL, de Kleijn DP, Hoefer IE, Pasterkamp G. Soluble ST2 levels are not associated with secondary cardiovascular events and vulnerable plaque phenotype in patients with carotid artery stenosis. Atherosclerosis 2013; 231: 48-53.

15 Demyanets S, Speidl WS, Tentzeris I, Jarai R, Katsaros KM, Farhan S, Krychtiuk KA, Wonnerth A, Weiss TW, Huber K, Wojta J. Soluble ST2 and interleukin-33 levels in coronary artery disease: relation to disease activity and adverse outcome. PLOS ONE 2014; 9: e95055.

16 Bartunek J, Delrue L, Van Durme F, Muller O, Casselman F, De Wiest B, Croes R, Verstreken S, Goethals M, de Raedt H, Sarma J, Joseph L, Vanderheyden M, Weinberg EO. Nonmyocardial production of ST2 protein in human hypertrophy and failure is related to diastolic load. J Am Coll Cardiol 2008; 52: 2166-2174.

17 Sanada S, Hakuno D, Higgins LJ, Schreiter ER, McKenzie AN, IL-33 Lee RT, and ST2 comprise a critical biomechanically induced and cardioprotective signaling system. J Clin Invest 2007; 117: 1538-1549.

18 Weinberg EO, Shimpo M, De Keulenaer GW, MacGillivray C, Tominaga S, Solomon SD, Rouleau JL, Lee RT. Expression and regulation of ST2, an interleukin-1 receptor family member, in cardiomyocytes and myocardial infarction. Circulation 2002; 106: 2961-2966.

19 Willems S, Hoefer I, Pasterkamp G. The role of the Interleukin 1 receptor-like 1 (ST2) and Interleukin-33 pathway in cardiovascular disease and cardiovascular risk assessment. Minerva Med 2012; 103: 513-524.

20 Miller AM, Xu D, Asquith DL, Denby L, Li Y, Sattar N, Baker AH, Mclnnes IB, Liew FY. IL-33 reduces the development of atherosclerosis. J Exp Med 2008; 205: 339-346.

21 Liew FY, Pitman NI, Mclnnes IB. Disease-associated functions of IL-33: the new kid in the IL-1 family. Nat Rev Immunol 2010; 10: 103-110.

22 Mancia G, Fagard R, Narkiewicz K, Redon J, Zanchetti A, Bohm M, Christiaens T, Cifkova R, De Backer G, Dominiczak A, Galderisi M, Grobbee DE, Jaarsma T, Kirchhof P, Kjeldsen SE, Laurent S, Manolis AJ, Nilsson PM, Ruilope LM, Schmieder RE, Sirnes PA, Sleight P, Viigimaa M, Waeber B, Zannad F, Redon J, Dominiczak A, Narkiewicz K, Nilsson PM, Burnier M, Viigimaa M, Ambrosioni E, 
npg

IL-33/sST2 and subclinical atherosclerosis

I Ates et al

518

Caufield M, Coca A, Olsen MH, Schmieder RE, Tsioufis C, van de Borne P, Zamorano JL, Achenbach S, Baumgartner H, Bax JJ, Bueno H, Dean V, Deaton C, Erol C, Fagard R, Ferrari R, Hasdai D, Hoes AW, Kirchhof P, Knuuti J, Kolh P, Lancellotti P, Linhart A, Nihoyannopoulos P, Piepoli MF, Ponikowski P, Sirnes PA, Tamargo JL, Tendera M, Torbicki A, Wijns W, Windecker S, Clement DL, Coca A, Gillebert TC, Tendera M, Rosei EA, Ambrosioni E, Anker SD, Bauersachs J, Hitij JB, Caulfield M, De Buyzere M, De Geest S, Derumeaux GA, Erdine S, Farsang C, Funck-Brentano C, Gerc V, Germano G, Gielen S, Haller H, Hoes AW, Jordan J, Kahan T, Komajda M, Lovic D, Mahrholdt H, Olsen MH, Ostergren J, Parati G, Perk J, Polonia J, Popescu BA, Reiner Z, Ryden L, Sirenko Y, Stanton A, Struijker-Boudier H, Tsioufis C, van de Borne P, Vlachopoulos C, Volpe M, Wood DA. 2013 ESH/ESC guidelines for the management of arterial hypertension: the Task Force for the Management of Arterial Hypertension of the European Society of Hypertension (ESH) and of the European Society of Cardiology (ESC). Eur Heart J 2013; 34: 2159-2219.

23 Friedewald WT, Levy RI, Fredrickson DS. Estimation of the concentration of low-density lipoprotein cholesterol in plasma, without use of the preparative ultracentrifuge. Clin Chem 1972; 18: 499-502.

24 Seki K, Sanada S, Kudinova AY, Steinhauser ML, Handa V, Gannon J, Lee RT. Interleukin-33 prevents apoptosis and improves survival after experimental myocardial infarction through ST2 signaling. Circ Heart Fail 2009; 2: 684-691.

25 Dieplinger B, Mueller T. Soluble ST2 in heart failure. Clin Chim Acta 2015; 443 : 57-70.
26 Coglianese EE, Larson MG, Vasan RS, Ho JE, Ghorbani A, McCabe EL, Cheng S, Fradley MG, Kretschman D, Gao W, O'Connor G, Wang TJ, Januzzi JL. Distribution and clinical correlates of the interleukin receptor family member soluble ST2 in the Framingham Heart Study. Clin Chem 2012; 58: 1673-1681.

27 Ho JE, Larson MG, Ghorbani A, Cheng S, Vasan RS, Wang TJ, Januzzi JL Jr. Soluble ST2 predicts elevated SBP in the community. J Hypertens 2013; 31: 1431-1436 discussion 1436.

28 Demyanets S, Kaun C, Pentz R, Krychtiuk KA, Rauscher S, Pfaffenberger S, Zuckermann A, Aliabadi A, Groger M, Maurer G, Huber K, Wojta J. Components of the interleukin-33/ST2 system are differentially expressed and regulated in human cardiac cells and in cells of the cardiac vasculature. J Mol Cell Cardiol 2013; 60: $16-26$.

29 Chen WY, Hong J, Gannon J, Kakkar R, Lee RT. Myocardial pressure overload induces systemic inflammation through endothelial cell IL-33. Proc Natl Acad Sci USA 2015; 112: 7249-7254

30 Kakkar R, Hei H, Dobner S, Lee RT. Interleukin 33 as a mechanically responsive cytokine secreted by living cells. J Biol Chem 2012; 287: 6941-6948.

31 Moussion C, Ortega N, Girard JP. The IL-1-like cytokine IL-33 is constitutively expressed in the nucleus of endothelial cells and epithelial cells in vivo: a novel 'alarmin'? PLOS ONE 2008; 3: e3331.

Hypertension Research 